\title{
NIVELES DEL LENGUAJE VALORATIVO
}

\section{I}

En el presente ensayo aplicaré ciertas categorías, usadas en el análisis del lenguaje científico, al análisis del lenguaje valorativo y pondré en discusión las especies de ética que resultarian de esa aplicación. Tomaré las categorías en cueśtión del lúcido análisis de Hempel en Fundamentals of Concept Formation in Empirical Science, ${ }^{1}$ y preguntaré cuál sería el desarrollo de la ética si se la considerara como una ciencia empírica y si tuviera lugar en ella una formación de conceptos como en las ciencias naturales. El resultado de nuestra discusión será algo un tanto obvio pero que rara vez se comprende en ética: a saber, que el desarrollo de la ética en el futuro requiere la creación de una estructura formal $y$ sistemática de referencia.

Según Hempel, una ciencia es un sistema teorético aplicado a un conjunto de observaciones. En otras palabras, una ciencia consta de tres partes: 1) un sistema teorético; 2) un conjunto de acontecimientos observados; 3) la conexión entre ambos, denominada indistintamente: la aplicación del sistema teorético a las observaciones, la interpretación de las observaciones en términos del sistema teorético, o la subsunción de las observaciones bajo del sistema. La ciencia no es ni el puro sistema teorético, ni las puras observaciones, sino tan sólo su combinación. Así, ni las matemáticas utilizadas en física, ni las observaciones realizadas por los físicos, sino tan sólo su combinación, la interpretación de las observaciones a la luz de las matemáticas, constituye la ciencia física. Hempel compara una teoría científica a "una compleja red espacial: los nudos representan a los términos, mientras que las fibras que los conectan corresponden, en parte, a las definiciones y, en parte, a las hipótesis fundamentales y derivadas incluídas en la teoría. Todo el sistema flota, por así decirlo, sobre el plano de la observación y está anclado en él por las reglas de la interpretación. Estas podrían representarse por cuerdas que no forman parte de la red, pero que enlazan ciertos puntos de ésta con lugares específicos del plano de observación. En virtud de esas conexiones interpretativas, Ia red puede funcionar como una teoría científica: desde ciertos datos observados, podemos ascender, por la vía de una cuerda interpretativa, hasta algún punto de la red teorética y proseguir luego, por la vía de las definiciones y de las hipótesis, hasta otros puntos, de los que nos permite descender al plano de la observación otra cuerda interpretativa. De esta manera, una teoría interpretada hace posible inferir la aparición de ciertos fenómenos que

1 Carl G. Hempel, Fundamentals of Concept Formation in Empirical Science, Chicago, 1952. 
pueden describirse en términos de observación". ${ }^{2}$ En otras palabras, el sistema teorético determina, por la interpretación empírica, la correlación entre las observaciones, y todo el conjunto de los datos observados recibe su unidad por las correlaciones ínsitas en el sistema teorético.

Para poder aplicarse, el sistema formal tiene que ser lo suficientemente preciso y detallado para dar cuenta de las correlaciones de los datos empíricos. Un conjunto de principios puramente generales no constituye un sistema aplicable; por ejemplo, el término clave del neo-vitalismo, "entelequia", carece de lo que Hempel llama alcance empírico. En cambio, un término como "gravitación universal" sí posee este alcance, pues "decir que las regularidades del movimiento planetario pueden explicarse por medio del concepto de gravitación universal, es una manera elíptica de afirmar que estas regularidades pueden explicarse por medio de la teoría formal de la gravitación, junto con la interpretación usual de sus términos". ${ }^{3}$ La razón de que el concepto "entelequia" carezca de significación empírica y el concepto "gravitación universal" la posea, es que el primero carece de significación teorética o sistemática y el segundo la posee; es decir, que el segundo permite "el establecimiento de principios ... explicativos bajo la forma de leyes generales o teorías" ${ }^{4}$ y es el centro de una red de relaciones formales. Elaborar conceptos con significación o alcance empírico es relativamente sencillo; "pueden fácilmente definirse en cualquier número, pero la mayoría de ellos carecerán de toda utilidad para propósitos sistemáticos... Es... el descubrimiento de sistemas de conceptos con alcance teorético lo que hace progresar la comprensión científica". ${ }^{5}$

La triple estructura de la ciencia se deriva de tres especies de definiciones, a saber: 1) análisis empírico o definición real, que Hempel (como Kant) llama explicación o exposición (Erklärung); 2) análisis conceptual, que Hempel (como Kant) llama definición analítica; y 3) interpretación ficticia, que Hempel llama definición nominal y Kant definición sintética. ${ }^{6}$ La exposición empírica (definición real) y el análisis significativo (definición analítica) producen explicaciones empíricas preliminares, pero no introducen las nuevas expresiones de que se precisa cuando se requieren ambas significaciones, la sistemática y la empírica. Esto sólo se lleva al cabo por la definición nominal o por las interpretaciones ficticias. Estas "no se introducen por medio de definiciones ... basadas en datos observables; de hecho, no se introducen por ningún proceso fragmentario que les asignara su significado individualmen-

2 C. G. Hémpel, op. cit., pág. 36. La analogía de Hempel trae a la mente un símil: La totalidad de una ciencia es como el aparejo flotante utilizado para la perforación del petróleo submarino. La plataforma es la teoría, el terreno subacuático los datos, los taladros las conexiones interpretativas. Los taladros exploran el subsuelo en puntos que no guardan conexión entre sí y, mediante la maquinaria de la plataforma, esos puntos quedan conectados con sentido.

3 op. cit., pág. 40.

4 op. cit., pág. 46.

5 op. cit., págs. 46 sigs.

6 op. cit., págs. 8 sigs. 
te. Más bien, las interpretaciones utilizadas en una teoría se introducen conjuntamente, por así decirlo, estableciendo un sistema teorético formulado en términos que les son propios y dándole a ese sistema una interpretación experimental".7 Así, "la geometría pura no formula aserto alguno acerca de las propiedades espaciales y de las relaciones de los objetos en el mundo físico. A partir de un sistema de geometría pura se obtiene una geometría física, es decir, una teoría que trata de los aspectos espaciales de los fenómenos físicos, dando una interpretación específica de aquélla en términos físicos. Así, por ejemplo, para obtener la correspondencia física de la geometría pura euclidiana, los puntos pueden interpretarse como semejantes a pequeños objetos físicos ...; una línea recta puede figurarse por la trayectoria de un rayo luminoso en un medio homogéneo; la congruencia entre intervalos por una relación física caracterizable en términos de coincidencias entre varas rígidas; etc. Esta interpretación convierte los postulados y teoremas de la geometría pura en proposiciones de la física". ${ }^{8}$

Existe, así, una diferencia fundamental entre la parte empírica y la parte teorética de una ciencia. La parte empírica describe fenómenos y, a lo sumo, proporciona definiciones generales, pero no una conexión universal; la parte teorética, en cambio, constituye una pauta de conexiones universales "a la que se conforman los fenómenos individuales". 9 Doquiera se encuentre una definición quẻ constituya una pauta de esta especie, será ésta tan general que parecerá enteramente alejada y desconectada de lo empírico. Por eso es tan sorprendente que Newton haya caído en la cuenta de la ley de la gravitación cuando le cayó una manzana en la cabeza.

El desarrollo de la ciencia, desde el análisis empírico y el análisis significativo hasta las construcciones teoréticas, se relaciona con el progreso que va del lenguaje cotidiano al lenguaje técnico y al lenguaje teorético o sistemático que, posteriormente, en las ciencias naturales, será matemático. "Los estadios iniciales de la investigación científica están establecidos en el vocabulario del lenguaje cotidiano. Con todo, el crecimiento de una disciplina científica implica siempre el desarrollo de un sistema de conceptos especializados, más o menos abstractos, de una correspondiente terminología técnica." ${ }^{10}$ Los términos técnicos "son sumamente abstractos y guardan escasa semejanza con los conceptos concretos que utilizamos para describir los fenómenos de nuestra experiencia cotidiana".11 Los conceptos utilizados en un sistema teorético son "ficticios" y carecen de toda conexión con la realidad empírica. "Puede concebirse un sistema teorético como una teoría en forma axiomática que no ha sido interpretada y que se caracteriza por: 1) un conjunto específico de tér-

7 op. cit., pág. 32.

8 op. cit., pág. 34.

0 op. cit., pág. 1 .

10 ibid.

11 op. cit., pág. 21. 
minos primitivos ...2) un conjunto de postulados, hipótesis primitivas o básicas; de ellas se obtienen otros enunciados de la teoría por deducción lógica... La geometría euclidiana... en cuanto 'geometría pura', es decir, en cuanto sistema axiomático no interpretado, es lógicamente del todo independiente de su interpretación en física y de su uso en navegación, topografía, etc." 12

Con la generalización creciente, desaparece la exactitud y, en cierto sentido, la precisión. Pero lo que distingue al lenguaje sistemático del cotidiano y conceptual es que, mediante la significación sistemática de sus términos, el lenguaje sistemático añade a su carácter general un nuevo género de precisión que permite el establecimiento de "leyes mucho más sutiles y precisas" 13 que las que son posibles en el análisis empírico. Así, en el lenguaje empírico puede caracterizarse el aire como "una mezcla en proporciones específicas de oxígeno, nitrógeno y gases inertes". ${ }^{14}$ Pero sólo las definiciones del oxígeno, del nitrógeno y de los gases inertes en términos de la tabla de los elementos, ${ }^{15} \mathrm{y}$ de los últimos en términos de leyes quánticas, hacen posible el establecimiento preciso de las leyes acerca del aire.

El lenguaje científico tiene, así, tres niveles: 1) lenguaje empírico, que describe situaciones en términos cotidianos; 2) lenguaje técnico, que puede ser de dos clases: $a$ ) análisis conceptual, $b$ ) interpretación de conceptos en términos de un sistema teorético (nunca se subsumen "hechos" sino conceptos, "casos ideales"); y 3) el sistema teorético mismo, lenguaje sistemático, que no describe ninguna situación pero se aplica a situaciones y las ordena autónoma y normativamente en un todo, produciendo así la estructura empírico-teorética total que constituye la ciencia. Para usar del ejemplo de la física: ella 1) constituye una descripción empírica de situaciones físicas, una definición real de acontecimientos físicos que culmina en conceptos empíricos; $2 a$ ) es un análisis de esos conceptos, que los pone a prueba de la física práctica y experimental; $2 b$ ) es física teorética, subsunción de los resultados de $2 a$ ) bajo 3); aquí los acontecimientos físicos se definen de manera autónoma por una definición nominal, según la pauta puramente formal 3), como "matemáticas para físicos". Este último lenguaje, mediante el proceso $2 b$ ), sirve de pauta para el lenguaje empírico-analítico $2 a$ ) y lo modela de nuevo.

Pasemos ahora al lenguaje valorativo. Tendríamos, de manera correspondiente: 1) descripción empírica de situaciones de valor, definición real del valor en términos de uno o varios rasgos de la situación, que culmina en conceptos empíricos de valor; $2 a$ ) análisis de esos conceptos, que los pone a prueba en la ética práctica y experimental (casuística); $2 b$ ) ética teorética, subsunción de los resultados de $2 a$ ) bajo 3); aquí el valor se define de manera autónoma por una definición nominal, según una pauta axiológica formal 3 ),

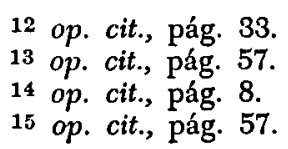


como "axiología para éticos". Este lenguaje, mediante el proceso $2 b$ ); sirve de pauta al lenguaje empírico-analítico 2 a) y lo modela de nuevo.

Ni el lenguaje valorativo 3) ni el $2 b$ ) existen aún en un grado importante. Por ello la ética actual no es una ciencia. Consiste en su mayor parte en los niveles 1) y $2 a$ ); sus conceptos carecen de significación empírica y sistemática; se asemejan más a la noción de "entelequia" que a la de "gravitación universal". Por otra parte, de lo dicho se sigue que la ética sería una ciencia tan pronto como obtuviéramos el nivel 3). Desaparecería la actual arbitrariedad de sus determinaciones valorativas empíricas -como placer, propósito, interés y otras similares, en tanto determinantes supuestamente valorativosy tomaría su lugar una definición del valor que tendría significación tanto sistemática como empírica.

\section{II}

La transformación de la filosofía moral en una ciencia supondría -como supuso la transformación de la filosofía natural en ciencia- la creación de una estructura formal de referencia y su aplicación a los fenómenos de valor. En el estado actual de la ética y de la teoría del valor, pueden concebirse tres estructuras formales de ese tipo: la semiótica, las matemáticas y la lógica.

\section{a) Análisis semiótico}

El análisis semiótico del lenguaje valorativo empírico se concentra en la configuración del habla: signo, significación, significado. Es algo menos abstracto de lo que sería un análisis del lenguaje valorativo de primer nivel en términos de su estructura lógica. Pues el lenguaje considerado semióticamente aún forma parte de la situación en que ocurre, aunque esta situación se ha abstraído y estilizado ahora considerablemente hasta que se ha reducido a un esquema de tres relaciones: las que median entre el que habla, el signo y el significado o término de referencia del signo. Se elimina así, en gran medida, la arbitrariedad en la selección de los elementos situacionales, inherente al lenguaje valorativo del primer nivel. ${ }^{16}$ Queda, con todo, la cuestión acerca de la pertinencia del análisis semiótico para la definición del carácter ético del lenguaje analizado. No puede resolverse esta cuestión en el segundo nivel de análisis y, por ende, en tanto que el análisis semiótico, es análisis de segundo nivel, padece de la misma insuficiencia, es decir, de la misma arbitrariedad definitoria, que el lenguaje de primer nivel. Charles Morris, aunque se ha ocupado del aspecto valorativo de la semiótica, ${ }^{17}$ no ha logrado

16 Con todo, cuando se define el lenguaje semiótico en términos de psicología conductivista desaparece està ventaja y la semiótica regresa al primer nivel.

17 Signs, Language and Behavior, New York, 1946; págs. 79 sigs.; Ray Lepley ed., Value: A Cooperative Inquiry, New York, 1949; págs. 211 sigs. Véase también Ray Lepley ed., The Language of Value, New York, 1955. 
definir aún el valor en términos de su sistema. Con todo, existe la posibilidad de un lenguaje semiótico en un tercer nivel. ${ }^{18}$ Habría de ser un lenguaje que no se refiriese al lenguaje valorativo de primer nivel ni lo interpretara, sino que le sirviera de sistema, de tal suerte que el lenguaje empírico quedara subsumido en él. Supongamos un sistema semiótico consistente, autosuficiente $y$ coherente, que proponga las relaciones formales que convierten a los signos apreciativos, a los significados en términos valuados y a las significaciones en significaciones valorativas; en otras palabras: ciertas configuraciones ínsitas en el sistema semiótico aparecerían como el conjunto de reglas aplicable a los fenómenos morales y capaz de analizar tanto las situaciones morales como los conceptos que se refieren a ellas. Entonces podría asignársele un lugar definitivo dentro del sistema a cada situación de valor o, más bien, a cada proposición referente a ella, y se cumplirían los requisitos de un lenguaje valorativo de tercer nivel. Este tipo de lenguaje sería diferente del lenguaje semiótico de segundo nivel. No sólo analizaría el lenguaje empírico sino que le serviría de pauta. El lenguaje empírico se subsumiría en él -se dividiría, ordenaría y moldearía de nuevo de acuerdo con la pauta sistemática- justo como los términos del lenguaje empírico se moldean y forman de nuevo, pero no se eliminan, en el lenguaje de la ciencia matemática. Mientras en el análisis semiótico de segundo nivel el punto de partida es el lenguaje empírico que se analiza sin alterar sus términos y relaciones, su pauta global- el punto de partida del análisis de tercer nivel es el sistema de tercer nivel mismo, y el lenguaje empírico no es para él más que una materia prima que no se disgrega en modo alguno en el proceso de análisis. El resultado es ininteligible para cualquiera menos para el experto en el lenguaje de tercer nivel -lo que significa, precisamente, que hemos dejado atrás el lenguaje empírico. El procedimiento para alcanzar este punto sería: primero, definir el valor en términos de semiótica, segundo, aplicar esta semiótica valorativa a los conceptos morales. Mientras no se establezca un sistema semejante, todo lo que puede hacer el análisis semiótico -y éste es ya un importante servicio- es afinar el lenguaje valorativo $y$, con ello, la observación de los fenómenos morales. Pero no puede acotar el dominio de la moralidad ni definirlo.

\section{b) Análisis matemático}

Por análisis matemático del lenguaje valorativo podemos entender, una vez más, dos cosas diferentes: análisis de segundo nivel o construcción de tercer nivel.

No hay ninguna razón para que las nociones fundamentales de la matemática no pudieran aplicarse a las de la ética. Podemos remontar el análisis matemático del lenguaje valorativo a Pitágoras y Platón, al cálculo de la jus-

18 Véase la sugerencia del autor en Ray Lepley ed., The Language of Value, New York, 1955. 
ticia de Aristóteles, a la Ethica more geometrico demonstrata de Spinoza, a la convicción de Locke acerca del carácter igualmente demostrable de la matemática y de la ética, al cálculo de Bentham, al "canon universal para computar la moralidad de cualquier acto" de Hutcheson, ${ }^{19}$ y a Rueff, ${ }^{20}$ Margenau, ${ }^{21}$ Northrop, ${ }^{22}$ Bertha B. Friedman, ${ }^{23}$ y Hermann Friedmann, ${ }^{24}$ para sólo mencionar algunos en nuestro tiempo. G. E. Moore coloca el "bien" al lado del número. ${ }^{25}$ Whitehead ha defendido convincentemente la combinación de ética y matemáticas, al mostrar la conexión que existe entre los patrones de organización y la concepción del bien. ${ }^{26}$ Es cierto que esto parece una aplicación de la lógica, más bien que de las matemáticas, al dominio del valor. El programa de largo alcance que Whitehead prevé para la lógica simbólica es, precisamente, un sistema valorativo:

Cuando, en el lejano futuro, el tema se haya desarrollado hasta llegar al examen de fórmulas que dependan de otras conexiones que las del espacio, el número y la cantidad; cuando haya ocurrido este desarrollo, sospecho que la Lógica Simbólica, es decir, el análisis simbólico de fórmulas con variables reales, llegará a ser el fundamento de la estética. A partir de esta fase procederá a la conquista de la ética y de la teología. 27

Pero este análisis de fórmulas puede llamarse también matemática. "Las matemáticas se están transformando ahora en el análisis intelectual de los tipos de fórmulas... Las matemáticas son la técnica más poderosa para el análisis de las relaciones de fórmulas." 28

Existen, empero, posibilidades de sistemas éticos más estrictamente matemáticas. Podría tomarse en consideración, por ejemplo, una aplicación del cálculo diferencial al proceso de crecimiento moral tal como ha sido descrito por Dewey o por Sartre, o una aplicación del análisis combinatorio a los métodos de acuerdo o desacuerdo ${ }^{29} \mathrm{o}$ bien, y aquí se ha realizado un intento

19 An Inquiry Concerning Moral Good and Evil, secc. III, xi, en Selby-Bigge, British Moralists, Oxford, 1897; I, págs. 110 sigs.

20 From the Physical to the Social Sciences, Baltimore, 1929; especialmente cap. XIV: interpretación de las teorías éticas tradicionales como "sistemas geométricos".

21 "Remarks" ( sobre ciencia ética), en The nature of Concepts. Their Interrelation and Role in Social Structure, Stillwater, Oklahoma, 1950. Igualmente: "Ethical Science", Scientific Monthly, 1949; pág. 290.

22 "Ethics and the Integration of Natural Knowledge", ibid. Igualmente: The Logic of the Social Sciences and the Humanities, New York, 1947.

23 Foundations of the Measurement of Values, New York, 1946.

24 Wissenschaft und Symbol, München, 1949.

25 Principia Ethica, págs. 110 sigs.

26 "Mathematics and the Good", The Philosophy of Alfred North Whitehead, P. A. Schilpp ed., Evanston, 1941.

27 "Remarks" en Ia sesión de la American Philosophical Association, Philosophical Review, vol. XLVI, 1937; pág. 186; The Philosophy of Alfred North Whitehead, pág. 324. 28 "Mathematics and the Good", op. cit., págs. 677 sigs.

29 Véase Robert S. Hartman, "Group Membership and Class Membership", Philosophy and Phenomenological Research, vol. XIII, 1953; n. 3. 
muy significativo, la aplicación de la "lógica de la relatividad" a la ética ${ }^{30} \mathrm{La}$ ética, si ha de ser un sistema, será un "espacio" en el moderno sentido de la palabra, y éste es, ciertamente, uno de sus aspectos fundamentales en cuanto ciencia. ${ }^{31}$ La geometría de los espacios no euclidianos es, por otra parte, un aspecto fundamental de las matemáticas. Lanz combina así la ética y las matemáticas de una manera fundamental. Su estudio sería una solución del problema del lenguaje valorativo si no se hubiera detenido a mitad del camino. No define el dominio de la ética de acuerdo con el sistema de coordenadas que traza, sino que más bien toma a la ética en su forma tradicional y simplemente analiza en sus propios términos sus arbitrarios conceptos empíricos y sus situaciones. No utiliza su sistema en el tercer nivel, como bien hubiera podido hacerlo, sino simplemente en el segundo. Su sistema no es propiamente un sistema matemático, sino una interpretación de lógica mediante las técnicas del análisis tensorial. Esta interpretación de lógica es altamente sugestiva y puede muy bien constituir una contribución importante al ajuste necesario de la lógica para hacerla aplicable a la ética. Pero, aụnque analiza el lenguaje ético de primer nivel, no logra integrar este lenguaje en el sistema. Más bien, se traza el sistema para mostrar que todas las teorías éticas tienen justificación tan sólo si se consideran desde un esquema de referencia superior que garantice su transformación recíproca. Lanz no utiliza su esquema de referencia para re-trazar las distintas éticas, para integrarlas y re-construirlas. Deja esta tarea para el futuro, como algo, dice con Luke, "que se requerirá de esta generación". ${ }^{32}$ La razón de que no logra hacerlo él mismo es que no llega a definir la superestructura que habrá de ser normativa para las distintas especies de ética. En otras palabras, no logra trazar una axiología que funcione. Su trabajo es similar al de G. E. Moore en tres respectos: a) no logra definir el valor; con todo, $b$ ) pone en claro su posición sistemática y c) es consciente de haber escrito los prolegómenos de una nueva ciencia.

\section{c) Análisis lógico}

Por análisis del lenguaje valorativo pueden entenderse, una vez más, dos cosas: análisis de conceptos valorativos o creación de interpretaciones valorativas. Lo primero sería análisis de segundo nivel y selectivo; lo segundo sería sistematización de tercer nivel y definitoria del lenguaje valorativo. Aquí aparece la diferencia entre análisis de segundo y de tercer nivel no sólo en la selección de los fenómenos en la realidad, sino también en la selección de los campos de acuerdo con la pauta aplicada, es decir, de acuerdo con la lógica. En otras palabras: cuando se aplica una pauta sistemática a un dominio feno-

30 Henry Lanz, In Quest of Morals, Stanford University Press, 1941.

31 Véase Robert S. Hartman, "The Moral Situation: A Field Theory of Ethics", Journal of Philosophy, vol. XLV, 1948; n. 11.

32 Lanz op. cit., pág. 163. 
ménico, no basta seleccionar un campo limitado dentro del marco de esa pauta; sino que la pauta, en sus axiomas y definiciones fundamentales ha de tener una afinidad con los fenómenos a los cuales se aplica. Pues la única ventaja de aplicar el sistema es precisamente que haya un sistema, y que puedan usarse sus relaciones formales para ordenar unos acontecimientos que de otro modo serían inconexos: tiene que haber una significación sistemática y empírica. Si se utilizara solamente una pequeña sección del sistema, que no tuviera conexión intrínseca con todo el sistema, quedaría defraudado el propósito de la construcción sistemática. La posibilidad de aplicación de las matemáticas a la naturaleza descansa, precisamente, en el hecho de que puede dársele una interpretación temporo-espacial a la noción fundamental de la matemática, al "número". Así, si ha de aplicarse un sistema a los fenómenos de valor, tendrá que haber una afinidad entre las nociones y relaciones fundamentales de ese sistema y el dominio del valor, y no simplemente una afinidad entre ciertas regiones aledañas del sistema y ciertas regiones de la ética. Podemos, por lo tanto, considęrar la diferencia entre análisis valorativo de segundo y de tercer nivel -entre análisis significativo de conceptos empíricos y normatividad sistemática aplicable a esos conceptos- como la diferencia entre la aplicación de configuraciones inesenciales y de configuraciones esenciales en el interior del sistema aplicado. Con respecto a la lógica, por análisis de segundo nivel se entendería análisis de lenguaje valorativo de primer nivel en términos de secciones de la lógica seleccionadas más o menos arbitrariamente; y por análisis de tercer nivel se entendería la subsunción del lenguaje de primer y segundo niveles bajo los axiomas y definiciones fundamentales de la lógica. Nos ocuparemos ante todo del primero, aunque, evidentemente no podamos tratar de él sin referirnos al segundo.

Pensamos, pues, en este punto, en interpretaciones del lenguaje valorativo de primer nivel en términos de algún o algunos campos elegidos de la lógica, como son las interpretaciones del lenguaje valorativo según una relación determinada, por ejemplo, la relación mejor que, ${ }^{33}$ o en términos de un modo o modalidad particular, por ejemplo, el imperativo ${ }^{34} u$ otros semejantes. Estas son aplicaciones selectivas y por ello arbitrarias, de la lógica a la moral, y no definiciones de la moral en términos de lógica. Podría establecerse, sobre otras relaciones o modos lógicos, cualquier número de sistemas éticos distintos, y la variedad de esos sistemas podría ser tan vasta como el contenido mismo de la lógica. Sólo la inventiva de los constructores de sistemas podría establecer sus límites. Se podría, por ejemplo, construir una ética sobre la modalidad de la imposibilidad, utilizando la definición de lo ético de James como "la vía de mayor resistencia"; o sobre la subsunción lógica, utilizando la noción de lo "apropiado" que usan Broad y otros; o, siguiendo a Jessup y

33 A. P. Brogan, "The Fundamental Value Universal", Journal of Philosophy, vol. XVI, 1919; n. 4; E. T. Mitchell, A System of Ethics, New York, 1950.

34 R. M. Hare, The Language of Morals, Oxford, 1952. 
a otros, se podría utilizar la noción misma de relación como prototipo de una ética situacional. Semejantes sistemas de ética serían (y son en la medida en que han sido escritos) un gran adelanto en la teoría ética. Una teoría empírica que se presta a tal interpretación manifiesta su inherente sistematicidad.

Pero esos sistemas aún son arbitrarios, así sea en un sentido muy limita. do, a saber, en el interior del sistema de la lógica. La utilización de la lógica misma para la interpretación del lenguaje ético no puede considerarse arbitraria; por lo contrario, parece muy natural, pues ¿para qué está la lógica sino para la interpretación del lenguaje? Existe arbitrariedad intra-sistemática mientras no se aclare sistemáticamente en qué respecto y sobre qué bases tenían que elegirse la relación, la modalidad y la función que se eligieron efectivamente por razones inherentes al sistema de lógica y, ciertamente también, a la naturaleza de la ética. Porque el campo lógico seleccionado no sólo ha de ser una región central y no meramente aledaña de la lógica -como la imperativa-, sino que también ha de corresponder a una región central y no meramente arbitraria de la ética. Así, el sistema ético basado en la relación de Brogan es el meliorismo; pero el meliorismo sólo es una especie de ética entre muchas. La relación lógica que sirva de base a un sistema de ética habría de ser lo suficientemente universal para incluir todos los sistemas éticos posibles, y no uno solo. Por otra parte, una ética basada en enunciados imperativos sólo da cuenta de una sección limitada del dominio moral. Lo mismo podría decirse de las otras especies de ética consideradas anteriormente. Hay, en suma, cuatro clases posibles de arbitrariedad en las combinaciones de la lógica y la ética, dispuestas en tres grados escalonados y en el siguiente orden de combinaciones: 1) combinaciones de campos lógicos aledaños con campos éticos aledaños; $2 a$ ) de campos lógicos aledaños con campos éticos centrales; $2 b$ ) de campos lógicos centrales con campos éticos aledaños; y 3 ) de campos lógicos centrales con campos éticos centrales. Sólo la última combinación representaría el auténtico sistema lógico de ética: una combinación, no de proposiciones seleccionadas, o de relaciones, modos o funciones seleccionadas con fenómenos morales y categorías éticas también seleccionadas, sino una combinación de fundamentos lógicos como tales con la naturaleza del bien. Combinaciones semejantes darían, por ejemplo, definiciones del "bien" en términos de la noción de función. proposicional, de vinculación ${ }^{35}$ de pertenencia a una clase, de valor de verdad, de extensión e intención. Una combinación tal de la ética y la lógica, si pudiera llevarse al cabo naturalmente y demostrarse como necesaria, constituiría un sistema de tercer nivel que debería cumplir los requisitos de un lenguaje valorativo normativo y dar cuenta

35 Para una sugerencia en esta dirección, véase: Robert S. Hartman, "The Analytic, the Synthetic, and the Good: Kant and the Paradoxes of G. H. Moore", op. cit. Para un tratamiento más explícito, véase: del mismo autor, "Value Propositions", en Ray Lepley ed., The Language of Value, New York, 1955, y "La Creación de una Etica Científica", Diánoia, vol. I, 1955; págs. 205 sigs. 
en sus propios términos de todo fenómeno de valor, así como de los análisis de esos fenómenos. Debería hacer eso por la razón de que el sistema sería coextensivo a ambos lenguajes, lógico y ético, y sería capaz, por ello mismo, de dar cuenta de manera sistemática de todo aquello de que dan cuenta esos lenguajes.

En este respecto, probablemente la combinación de lógica y ética es más eficàz que las demás combinaciones examinadas en este nivel: la combinación de la semiótica y la de la matemática con la ética. Con todo, es cierto para todas esas combinaciones que, a menos de conectar los fundamentos del sistema en cuestión con los fundamentos de la ética, la teoría resultante será arbitraria, aunque esa arbitrariedad sea de una especie superior, intra-sistemática o que concierna a selecciones efectuadas en el interior de un sistema $y$, por lo tanto, sea menos arbitraria que la arbitrariedad del primer nivel, la cual se esparce por todo el dominio de lo empírico.

Podría objetarse contra este requisito de combinar fundamento con fundamento, que es demasiado rígido y que no hay razón para que no pueda construirse una ética sistemática con cualquier material lógico. Podría sostenerse que las ciencias naturales se construyeron con cualquier material matemático, sin preocuparse por el carácter fundamental que tuvieran, dentro de las matemáticas, las secciones elegidas. Cabe decir que, para explicar los fenómenos naturales, se ha utilizado y se utilizan un sinnúmero de nociones matemáticas no relacionadas entre sí. Así, la astronomía utiliza el cálculo, ecuaciones integrales y diferenciales, y espacios no-euclideanos; la teoría eléctrica emplea los números complejos; la teoría quántica, el cálculo de matrices; la termodinámica el cálculo de probabilidades. Todas estas pautas se obtuvieron del cuerpo entero de las matemáticas más o menos a la ventura, y ateniéndose tan sólo a la estructura de los fenómenos de que había que dar cuenta. La filosofía natural podría sostenerse que nunca hubiera llegado a ser una ciencia si se hubiese limitado a aparejar tan sólo los fundamentos de las matemáticas con los fundamentos de la naturaleza. Para empezar, hubiera sido casi imposible decidir cuáles habrían de ser estos últimos.

Puede concederse que el desarrollo de la ciencia natural, mediante la combinación de esquemas matemáticos de referencia con fenómenos naturales, no procedió conscientemente del modo que requerimos para la ética. Pero bien puede igualmente sostenerse que sí procedió de esta manera. La originaria aplicación de la matemática a los fenómenos naturales, la cual dio lugar a la ciencia moderna, fue la intuición de que "la esencia de una cosa material consistía en ser res extensa", ${ }^{36}$ y la expresión algebraica del espacio en la geometría analítica de Descartes. Se llevó al cabo después de una profunda reflexión, sobre la naturaleza del pensamiento, por un lado, y sobre la esencia de la naturaleza, por el otro. Su segundo paso, la invención del cálculo por

${ }^{36}$ Edmund Husserl, Ideen zu einer reinen Phänomenologie und phänomenologischen Philosophie, \&9. 
Leibniz, fue precedido por una reflexión filosófica igualmente profunda sobre la naturaleza del pensamiento y de la realidad. Lo mismo vale, aunque en menor grado y en distinto sentido -es decir, el de restringir la investigación científica al mundo fenoménico- para Newton. Aún así, tratándose de un hombre que no inventaba hipótesis, Newton era singularmente metafísico. Una vez dados estos primeros pasos, el desarrollo de la matemática, tanto pura como aplicada, siguió su dirección original. Más aún, puede sostenerse que el impase actual de las ciencias naturales "en el umbral de la metafísica", para decirlo como Brühlmann, ${ }^{37}$ señala la necesidad de un nuevo punto de partida, de una nueva penetración del pensamiento especulativo en las honduras metafísicas del simbolismo -que puede conducir a aplicaciones matemáticas nuevas y enteramente inesperadas, como, por ejemplo, la aplicación de la teoría de los números a la teoría de las partículas materiales.

Pero incluso concediendo la objeción, hay una gran diferencia entre lo que podemos llamar, con G. E. Moore, "ética como ciencia sistemática" ${ }^{38}$ y la ciencia natural. En la ciencia natural no importaba si un esquema de referencia era seleccionado arbitrariamente o no, pues el experimento y la observación ponían a prueba la corrección de los resultados teoréticos. No hay una prueba semejante en la ciencia ética. Esta ciencia debe levantarse por la fuerza de su congruencia interna. Debe llevar consigo sus propias restricciones. Se trata de una situación semejante a la que debatió Kant con respecto a la ciencia metafísica. La diferencia entre la posibilidad de una ciencia natural y la de una ciencia metafísica consiste para Kant en que, en la ciencia natural, la intuición sensible restringe constantemente al entendimiento, mientras que en la ciencia metafísica la razón debe contener en sí misma sus propias restricciones. Sólo hay una restricción, basada en la naturaleza misma del pensamiento, a saber, la no contradicción. De ahí que la misión de la dialéctica trascendental consista en mostrar que las restricciones internas de la razón consisten en los varios tipos de contradicción. Nuestra situación es similar, sólo que apunta hacia una dirección positiva más que negativa. La ciencia natural matemática no ha menester de ninguna certidumbre especial interna, puesto que tiene una externa, su confirmación por la experiencia y el experimento. Pero la ciencia ética ha menester de una certidumbre especial interna y por ello requiere reglas más estrictas que la ciencia natural. Cualquier selección arbitraria de esquemas de referencia, provengan de la lógica, o de la semiótica, o de la matemática, o de cualquier otra fuente, es inadecuada para ella. Por esta razón todos los lenguajes de la ética en el segundo nivel son inadecuados, por más elaboradamente que estén o puedan estar desarrollados. Siempre estarían afectados por la carencia de necesidad, que sólo puede garantizarse mediante la combinación de fundamentos sistemáticos con fundamentos éticos. Así, un sistema de valor de tercer nivel ha

37 Otto Brühlmann, Physik am Tor der Metaphysik, München, 1935.

38 Principia Ethica., pág. 6. 
de ser inter-axiomático, con lo cual quiero decir que ha de conectar axiomás éticos con axiomas no éticos.

De esta exigencia surge una importante consecuencia. A menudo se argumenta contra la posibilidad de un sistema ético afirmando que nunca se podrá llegar a un acuerdo respecto de él. Pero una vez que tuviera ese sistema la certidumbre interna descrita y requerida como su presupuesto, no habría duda alguna para nadie de que ése era el sistema buscado, o, por lo menos uno muy semejante al ideal bajo esas circunstancias. De este modo, el acuerdo sería concomitante del sistema. En este respecto, no diferiría de un sistema científico. El escepticismo de quienes dudan por principio de un acuerdo acerca de los sistemas éticos resulta, por lo tanto, infundado. Está justificado para los análisis del lenguaje valorativo de primero y segundo nivel, pero no para la construcción del tercer nivel. Esta última es tan segura en su vía metódica como la matemática en la suya; $\mathrm{y}$, a menos de ser tan cierta como ella, no será un análisis de tercer nivel. A este respecto, Locke tenía razón cuando comparaba la demostrabilidad ética cọn la matemática.

\section{III}

Consideremos ahora más de cerca una axiología de tercer nivel.

Habría de consitir en un lenguaje valorativo sistemático, es decir, como ya va resultando claro, un sistema formal que no analizara sino que constituyera el lenguaje valorativo, definiéndolo autónomamente en sus propios términos. Es un sistema que define ciertos de sus axiomas como axiomas propios de la ética. De este modo define creadoramente el dominio de la ética. Este lenguaje de tercer nivel es independiente del lenguaje empírico y de sus usos. ${ }^{39}$ Aplica su propia terminología este lenguaje que le sirve de materia prima, justo como las descripciones de los procesos naturales y sus análisis sirven de materia prima a la interpretación matemática, y la matemática misma es independiente de esos materiales empíricos.

Lo dicho en las secciones precedentes acerca de la posible naturaleza de un lenguaje semejante resulta suficiente para comprender que son posibles en este nivel por lo menos tres de esos sistemas: el semiótico, el lógico y el matemático. Podría suceder que uno de esos tres resultara a la postre superior y constituyera, así, un cuarto nivel de análisis valorativo. Por ahora, el análisis lógico parece tener la mayor probabilidad de convertirse en un sistema de tercer nivel. Esto significaría que los términos, proposiciones y relaciones del lenguaje valorativo encontrarían su posịción sistemática mediante su referencia al sistema de la lógica, y que la ética y la lógica se combinarían, de un modo semejante a como se combinaron en la ciencia natural la física y la geometría. Podría ser que este desarrollo tuviera que esperar una expansión

30 Véase: C. G. Hempel, op. cit., pág. 49; Paul W. Kurtz, "Naturalistic Ethics and the Open Question", Journal of Philosophy, vol. LII, 1955; págs. 113 sigs. 
de la lógica, así como el correspondiente desarrollo de la física presupuso la expansión de la geometría desde los espacios euclideanos a los no-euclideanos. Por otra parte; es posible que los Lobachevsky y los Bolyai de la lógica ya hayan realizado su trabajo en las personas de Kant, Hegel, Husserl, Dewey y otros, ${ }^{40}$ así como Boole, Russell y Wittgenstein. La lógica cubre el dominio del pensamiento en su totalidad y cualquier dirección singular -incluso la matemática- es una selección arbitraria de la totalidad del campo de la lógica posible..1 Después de lo que hemos dicho pudieran utilizarse una o algunas de estas lógicas para elaborar los elementos lógicos de las teorías éticas, particularmente la teoría de G. E. Moore. Así, o bien como dice Susanne Langer, "los lógicos interesados en la ética serán probablemente los fundadores de la ética científica", ${ }^{42}$ o bien, como pudiera igualmente ocurrir, los fundadores serán los éticos interesados en la lógica. Una vez realizada su tarea, ya no existirá la división entre ética y lógica en el viejo sentido, puesto que no sería posible axiología alguna sin lógica, justo como actualmente no es posible física alguna sin matemática. Entonces, la ética tradicional -empirismo de primer nivel y análisis conceptual de segundo nivel- sólo tendrá un interés histórico.

Cualquiera que sea la forma final del sistema de la axiología, será fundamentalmente diferente de los niveles inferiores de análisis valorativo. Ante todo, ya no presentará los rasgos de arbitrariedad que encontramos en los niveles inferiores. Si aparecieran varios sistemas semejantes de tercer nivel, cada uno tendría que presentar el carácter de necesidad en sus propios términos, pues de otro modo no sería un sistema de tercer nivel. Es concebible que esos distintos sistemas se ocuparan de aspectos distintos, pero igualmente fundamentales, del dominio moral y ético, así como, actualmente, distintas especies de análisis dan cuenta de distintos fenómenos naturales en la teoría de la relatividad y la teoría de los quanta, pero con la misma necesidad y la misma significación fundamental para la física. Por la misma naturaleza de las cosas, no puede haber muchos sistemas semejantes de axiología, pues los requisitos que deben llenar estos sistemas son extremadamente severos. No sólo tendrá que ser congruente en sí mismo, cada sistema, también tendrá que concordar con las proposiciones fundamentales de la ética. Tendrá que dar cuenta de cualquier teoría ética, en cualquier nivel de análisis que sea, de la de Platón y la de Nietzsche, de la de James y la de Stevenson, de la de Hare y la de Hilliard, de la de Bentham y la de Moore, ${ }^{43}$ de la de Kant, la de

40 Véase: Ernst Manheim, Zur Logik des konkreten Begriffs, München, 1930; Bernad Mayo, The Logic of Personality, London, 1952.

41 Cf. B. Blanshard, The Nature of Thought, especialmente: I, págs. 629 sigs. y 640; II, págs. 356 sigs.

${ }_{42}$ The Practice of Philosophy, New York, 1930; págs. 213 sigs.

43 Si hay un sistema axiológico formal tendría que desarrollarse a partir de una definición nominal del "bien" (véase: supra nota n. 6). Esto no se opondría a la pretensión de Moore de que el "bien" que sólo se refiere a las definiciones empíricas y analíticas es 
Urban y la de Kierkegaard. Tendrá que hacerlo, no de modo general, sino específico. Con la misma precisión, tendrá que dar cuenta de los fenómenos morales mismos.

Pero aún no es suficiente todo esto: la formalidad y universalidad de la teoría significaría que en ella quedaría comprendido algo más que la mera aplicabilidad al dominio moral y a sus lenguajes. Tendría que ser una teoría general del valor, aplicable y normativa no sólo para la ética, sino para todos los campos del valor. Pues

si ha de haber una ciencia del valor, alguien tiene que formular las concepciones básicas que darán lugar a la ética, a la estética $y$, posiblemente a la economía, todo de una vez. Si el valor constituye realmente un campo definible, todas estas ciencias están relacionadas... Probablemente el sistema en su totalidad, una vez descubierto, no se parecerá a la actual teoría del valor más de lo que la astronomía se parece a su precursora, la astrología.44

Así, el nuevo sistema no sólo tendría que tomar en cuenta y relacionar entre sí todos los fenómenos y proposiciones de la ética, sino también los de todas las demás disciplinas que se ocupan del valor, y éste parece ser el campo entero llamado anteriormente "filosofía moral" y hoy "ciencia social" y "humanidades". Y tendría que hacer esto con la misma precisión con que la física correlaciona "la mecánica y la físico-química y la electrodinámica... mediante un sistema elaborado único". 45

Si esto es así, y si el nuevo sistema incluye las normas no sólo de la ética, sino de todas las demás ciencias valorativas, su lógica inherente -su alcance sistemático- deberá conducir a descubrimientos empíricos siempre nuevos

indefinible. La falacia naturalista sería, entonces, la confusión entre el tercer nivel y los demás niveles del discurso de valor, es decir, entre la axiología y sus aplicaciones. El "bien" de Moore sería más bien axiológico que ético, estético, o cualquier otro "bien" específico. La axiología misma sería la expansión sistemática de la definición nominal del "bien" y como tal, sería interpretativa de los niveles inferiores. Así, si "bien" se define como el predicado indicador de que el sujeto tiene ciertos predicados (véase: supra nota n. 35) sería un enunciado axiologico decir: "esto es bueno'. Significa "esto tiene las cualidades o relaciones X, Y, Z..." (véase Charles L. Stevenson, Ethics and Language, pág. 207). Pero decir "Juan es un hombre fundamentalmente bueno... tiene el más amable de los corazones" (ibid., pág. 29), seria un enunciado ético. Sería falacia naturalista decir: " "esto es bueno' significa tener un buen coraxón”, si por "bueno" se significa más bien el "bien" axiológico que el ético. La distinción de niveles en el lenguaje valorativo resuelve otras dificultades metodológicas, como en el caso del ejemplo de Stevenson: "cuando hay vino, mujeres y canto está mal no hacer algo malo" (ibid., pág. 84). El primer "mal" es axiológico, el segundo ético. O bien la frase: "cuanto mejor sea una conciencia, peor será, cuanto peor sea, mejor será" que significa que cuanto mejor sea una conciencia axiológicamente, es decir, cuanto más sensitiva sea, será peor éticamente, es decir, será más a menudo "una mala conciencia" en el sentido ético (siempre que se defina una "buena conciencia" como una conciencia sensitiva); y viceversa, cuanto menos sensitiva sea una conciencia, es decir, cuanto peor sea axiológicamente, "mejor" será moralmente, es decir, con tanta menor frecuencia será "una mala conciencia" en el sentido ético.

44 Susanne Langer, op. cit., pág. 209.

45 ibid. 
-su alcance empírico en los campos de realidad del valor-conforme se vayan encontrando nuevas aplicaciones del sistema a la realidad. Más aún, el sistema mismo se desarrollaría más y más, y eventualmente se dividiría en axiología pura y aplicada. Los axiólogos puros, como actualmente los matemáticos puros y los lógicos matemáticos, se dedicarían al desarrollo del sistema como tal, mientras que los tratadistas de axiología aplicada serían los futuros científicos sociales, psicólogos, sociólogos, economistas, científicos de la política-, así como los futuros científicos de la ética, de la estética y de la metafísica, cuyas ciencias serían precisamente, las aplicaciones del nuevo sistema a sus campos respectivos.

Existiría entonces en las ciencias sociales y en las humanidades la misma jerarquía de niveles de lenguaje que existe actualmente en las ciencias naturales. En el primer nivel habría descripciones empíricas de situaciones de valor, en el segundo nivel análisis conceptuales de estas descripciones, y en el tercer nivel el sistema autónomo que define en sus propios términos los distintos dominios del valor y funciona como su superestructura, del mismo modo como la matemática funciona como superestructura de la física y de otras ciencias naturales. Así como actualmente la matemática es el lenguaje de las ciencias naturales, la axiología será entonces el lenguaje de las ciencias sociales y humanas.

Robert S. Hartman

(trad. Luis Villoro)

Massachusetts Institute of Technology

Estados Unidos. 